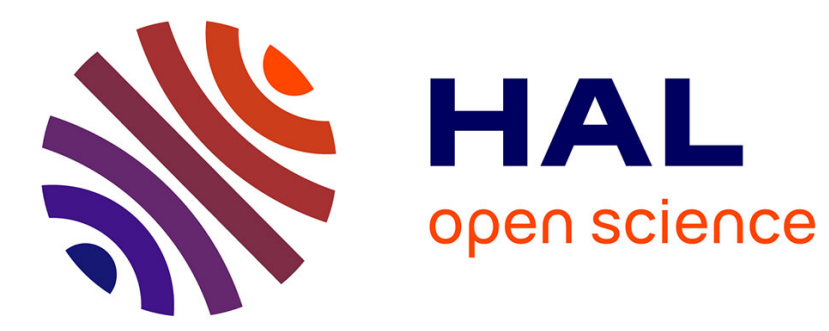

\title{
Why might planets and moons have early dynamos?
}

\author{
J. Monteux, A.M. M Jellinek, C.L. L Johnson
}

\section{To cite this version:}

J. Monteux, A.M. M Jellinek, C.L. L Johnson. Why might planets and moons have early dynamos?. Earth and Planetary Science Letters, 2011, 310 (3-4), pp.349 - 359. 10.1016/j.epsl.2011.08.014 . hal01636051

\section{HAL Id: hal-01636051 https://hal.uca.fr/hal-01636051}

Submitted on 11 Jan 2018

HAL is a multi-disciplinary open access archive for the deposit and dissemination of scientific research documents, whether they are published or not. The documents may come from teaching and research institutions in France or abroad, or from public or private research centers.
L'archive ouverte pluridisciplinaire $\mathbf{H A L}$, est destinée au dépôt et à la diffusion de documents scientifiques de niveau recherche, publiés ou non, émanant des établissements d'enseignement et de recherche français ou étrangers, des laboratoires publics ou privés. 
1. Why might Planets and Moons have Early Dynamos?

J. Monteux ${ }^{\mathrm{a}}$, A.M. Jellinek ${ }^{\mathrm{a}}$, and C.L. Johnson ${ }^{\mathrm{a}, \mathrm{b}}$
${ }^{3}$
${ }^{a}$ Department of Earth and Ocean Sciences, University of British Columbia, Canada.

\section{Abstract}

- Remanent crustal magnetization of martian and lunar crustal rocks plausibly

7 records magnetic fields related to core dynamos active during the first few hun-

s dred Myr of evolution of these bodies. Such early fields suggest that core dy-

9 namos may occur during or as a result of accretion. We investigate whether

10 the processes governing the segregation and sinking of metallic Fe after a large

11 impact can yield thermal conditions in the core that favour dynamo action on

12 growing planets. Depending on the sizes of the impactor and planet, as well as

13 the temperature-dependence of the viscosity, we identify conditions in which an

14 early transient core dynamo is possible. We also consider the effect of a molten

15 layer surrounding the protocore on the duration of this early dynamo. Our re-

16 sults suggest that dynamos can be initiated in bodies with a radius of $3500 \mathrm{~km}$

17 radius or greater under Earth-like conditions for ohmic dissipation in the core,

18 and in smaller bodies if a less restrictive critical magnetic Reynolds number

19 condition is applied. These dynamos may persist for several kyr to several Myr

2o depending on the heat transfer regime at the protocore-mantle boundary.

21 Key words: early dynamos; growing planets; meteoritic impacts; numerical

22 modelling. 


\section{Introduction}

Among the terrestrial planets and moons of the inner Solar System, Mars and possibly the Moon record evidence for early and short-lived (a few hundred Myr) internally generated magnetic fields. Mars Global Surveyor and Lunar Prospector magnetometer data (Acuña et al., 1999; Hood et al., 2003) confirm the presence of ancient magnetized crust on these bodies. Although later dynamo fields (beginning around or after $3.9 \mathrm{Ga}$ ) have been suggested for both bodies (Cisowski et al., 1983; Schubert et al., 2000), most evidence points to earlier dynamos, that ceased by $\sim 3.9 \mathrm{Ga}$ (Johnson and Phillips, 2005; Lawrence et al., 2008; Lillis et al., 2008; Garrick-Bethell et al., 2009). Moreover, recent analyses of paleomagnetism in angrite meteorites suggest that an early and internally generated dynamo occured within the angrite parent body (Weiss et al., 2008). Although there is increasing evidence for the occurrence of these transient dynamos, their origin and timing remain a major enigma. Here we investigate a plausible temporal link between early dynamos and the large impacts that characterize the late accretion histories of terrestrial bodies in our solar system. Assuming chondritic bulk compositions, these impacts can lead to extensive mantle melting, iron-silicate separation, iron segregation and ultimately core formation (Tonks and Melosh, 1992; Ricard et al., 2009; Monteux et al., 2009; Srámek et al., 2010). If dynamo action depends primarily on the rate of core heat loss (Buffett, 2002), then a crucial issue for explaining early dynamos is to understand the thermal states of both the impacted planet and the nascent core: Under what conditions will the resulting core cooling be sufficiently large to favor dynamo action?

Large meteorite impacts can govern the early stages of planetary formation.

Figure 1 shows a schematic temporal evolution of our impact-induced dynamo 
50

51

52

53

model from time $\boldsymbol{t}_{\mathbf{1}}$ (impact time) to time $\boldsymbol{t}_{\mathbf{5}}$ (dynamo is initiated). A collision between an impactor and an undifferentiated protoplanet leads to an increase in mass of the impacted body, the formation of an impact basin, the propagation of shock waves (Fig. 1, $\boldsymbol{t}_{\mathbf{1}}$ ) and a local spherical temperature increase, $\Delta T_{0}$, below the impact site (Fig. 1, $\boldsymbol{t}_{\mathbf{2}}$ ). All these processes are very fast compared to the following thermo-chemical re-equilibration. The temperature increase is constant within a spherical volume $V_{i c}$ called isobaric core with radius $R_{i c}$ and rapidly decreases away from it (Pierazzo et al., 1997; Senshu et al., 2002; Monteux et al., 2007). Assuming that meteorites impact at the escape velocity of the impacted body, this temperature increase scales with the size of the impacted planet and is superimposed on the initial mean temperature, $T_{0}$, of the planet before impact. For sufficiently large planets, the temperature increase can overcome the melting temperature of the metallic phase leading to metal-silicate separation (Monteux et al., 2009) (Fig. 1, $\boldsymbol{t}_{\mathbf{3}}$ ). The dense metallic phase sinks towards the center of the impacted body. How this occurs is debated. Proposed mechanisms include percolation through a solid mantle (Stevenson, 1990; Shannon and Agee, 1996) and negative diapirism in the form of cm-sized droplets in a magma ocean [e.g. Rubie et al. (2003)] to large mantle diapirs [e.g. Tonks and Melosh (1992); Senshu et al. (2002); Ricard et al. (2009)]. In addition, it has been proposed that metallic diapirs can sink towards the centre of the planet via fracturation and diking (Tonks and Melosh, 1992; Solomatov, 2000; Stevenson, 2003b) or viscous deformation (Honda et al., 1993; Monteux et al., 2009) depending on the thermal state of the planet. Here, we consider a simplified scenario in which a single large impact into an initially undifferentiated planet leads to a single Fe-diapir that sinks through an effectively viscous mantle at a Stokes velocity (Fig. 1, $\boldsymbol{t}_{\mathbf{4}}$ ). 
Whether convection and dynamo generation can occur in a liquid iron protocore depends on the temperature difference between the protocore and the surrounding mantle. A protocore sufficiently hotter than the surrounding silicate mantle is one way to explain the presence of an early dynamo on terrestrial planets and moons. Previous studies have examined the following possible causes of an early hot protocore and dynamo: radioactive heating (Yoshino et al., 2003; Williams and Nimmo, 2004; Nimmo, 2009), strong cooling by mantle convection (Nimmo and Stevenson, 2000; Stegman et al., 2003; Breuer and Spohn, 2003; Elkins-Tanton et al., 2005) or impact heating (Monteux et al., 2009; Reese and Solomatov, 2010). Here we focus on whether the thermal conditions resulting from a single large impact and the subsequent differentiation event are also favourable to dynamo initiation. In this case, the temperature difference between the protocore and the overlying mantle depends on the thermal evolution of the metallic diapir as it sinks through a colder mantle towards the centre of the planet (Fig. 1, $\boldsymbol{t}_{\mathbf{5}}$ ). Because the heat content and buoyancy of the diapir depend on its volume, one critical parameter will be the radius of the metallic diapir $R_{F e}$. An additional potentially important contribution to the initial core-mantle temperature difference will be the extent to which the gravitational potential energy released on sinking is converted into heat in the metallic diapir and in the surrounding mantle via viscous dissipation. The importance of this effect, and where it occurs (i.e., primarily in the diapir or surrounding mantle), will depend critically on the strongly temperature-dependent viscosity $\eta(T)$ of the rocks composing the protoplanetary mantle. For impacted planets of given radii $R$ we use the numerical model of Monteux et al. (2009) to identify $R_{F e}-R-\eta(T)$ conditions favoring dynamo action. In addition, we investigate the dependence of the dynamo duration on the protocore size, and on conditions that lead to melting of the overlying mantle material. 


\section{Thermo-chemical model}

\subsection{Initial state}

The initial structure and thermal state of a growing planet depends particularly on the characteristics of its accretion (Safronov, 1978; Kaula, 1979). Indeed, differentiated terrestrial planets probably formed rapidly from accumulation of chondritic material (Agee, 1997). In more detail, during accretion, heating driven by a combination of the dissipation of impact energy and the decay of short lived radionuclides such as ${ }^{26} \mathrm{Al}$ and/or ${ }^{60} \mathrm{Fe}$ (Senshu et al., 2002; Yoshino et al., 2003; Monteux et al., 2007) increases the mean internal temperature and gives rise to a radial temperature gradient that depends on the accretion rate relative to the rate of radiative cooling to space. Where this growth rate is very high in comparison to surface cooling, this heating can ultimately cause partial or complete melting (Yoshino et al., 2003) and extensive internal differentiation. In contrast, where the growth rate is very low in comparison to surface cooling, little additional heating occurs (Wood et al., 2006) and the growing planet remains an undifferentiated mechanical mixture of the accreted impactors. Hence, depending on its growth rate, the frequency of impacts and the size of the impactors, a protoplanet can potentially grow to a Mars size radius in $\approx 1$ Myr without undergoing any significant episodes of melting and any subsequent global differentiation processes (Senshu et al., 2002). Short-lived isotopic constraints restrict the timing of core formation on terrestrial planets to be within the first tens of Myr following accretion (Kleine et al., 2002; Yin et al., 2002; Touboul et al., 2007). From these models, the martian core formation occurred faster (within $30 \mathrm{Myr}$ ) than the Earth's core formation (within $50 \mathrm{Myr}$ ). Consequently, between 1Myr (the growing planet reaches a Mars size radius with no or little differentiation) and 50 Myr (the core is fully formed), large impacts can lead to episodic large differentiation events. 
Whether an impact onto an undifferentiated planet leads to melting and core formation depends critically on the fraction $\gamma$ of the initial kinetic energy $E_{k i n}$ of the impactor that is dissipated within the planet itself as a result of work done by shock waves (Tonks and Melosh, 1992; Monteux et al., 2009). $E_{k i n}$ is proportional to the impact velocity $v_{i m p}^{2}$, which can be decomposed into two contributions:

$$
v_{i m p}^{2}=v_{e s c}^{2}+v_{\infty}^{2}
$$

Here, $v_{e s c}$ is the escape velocity of the impacted planet and $v_{\infty}$ is the velocity of the impactor at a distance much greater than that over which the gravitational attraction of the impacted planet is important. A conservative and well-constrained estimate of $E_{k i n}$ is possible if we take $v_{\infty}=0$ and assume $v_{i m p}=v_{e s c}$. We note that in the case of an impact on a moon orbiting around a larger central body, the gravitational attraction of the central body can increase this impact velocity and we address this issue in section 5. Laboratory experiments and modelling studies suggest that the energy released within the planet is mainly dissipated by frictional heating and melting within a spherical region (an "isobaric core") with a volume $V_{i c}$ that is at most 3 times larger than the volume of the impactor $V_{i m p}$ (O'Keefe and Ahrens, 1977; Croft, 1982; Pierazzo et al., 1997; Senshu et al., 2002). Within this region the temperature increases uniformly from $T_{0}$ to $T_{0}+\Delta T_{0}$ where $T_{0}$ is the temperature of the impacted body before the impact and $\Delta T_{0}$ is the temperature increase due to impact heating. Experiments suggest also that there is an additional thin spherical shell of damaged and heated material outside the isobaric core. Assuming 
that the work done by shock waves in this shell causes no melting, the excess temperature $\Delta T_{0}$ decreases rapidly and smoothly with distance $r$ across the shell as approximately $\Delta T_{0}\left(R_{i c} / r\right)^{m}$. Following Senshu et al. (2002), and fitting the decay of peak pressure with distance away from the edge of the isobaric core $m \approx 4.4$ (Monteux et al., 2007). Hence, the amount of energy $E_{\text {ext }}$ dissipated as heat outside the isobaric core is:

$$
E_{\text {ext }}=2 \pi \overline{\rho_{0} C_{p}} \int_{0}^{\pi} \int_{R_{i c}}^{+\infty} \Delta T_{0}\left(\frac{R_{i c}}{r}\right)^{m} r^{2} \sin \theta d \theta d r
$$

with $\theta$ the zenith angle from the impact site and $\overline{\rho_{0} C_{p}}$ is the average specific heat of the impacted body. For the axisymmetric geometry of the isobaric core, the previous equation can be reduced to:

$$
E_{\text {ext }}=\overline{\rho_{0} C_{p}} V_{i c} \Delta T_{0}\left(\frac{3(2 m-5)}{2(m-3)(m-2)}\right)
$$

Assuming that $v_{i m p}=v_{e s c}\left(\right.$ Kokubo and Ida, 1996) and that $V_{i c}=3 V_{i m p}$ (Senshu et al., 2002), a balance between the kinetic energy delivered to heat the growing planet $\left(\gamma E_{k i n}\right)$ and the energy used to heat up and melt the isobaric core and to heat up the surrounding material without melting is

$$
\frac{4 \pi}{9} \gamma G \rho_{0}^{2} V_{i c} R^{2}=\overline{\rho_{0} C_{p}} \Delta T_{0} V_{i c}+E_{e x t}+V_{i c}\left(\rho_{F e} f_{0} L_{F e}+\rho_{S i}\left(1-f_{0}\right) L_{S i}\right) .
$$

After some algebra we obtain the temperature increase at the impact site as a function of the impacted planet radius (Monteux et al., 2007)

$$
\Delta T_{0}=\frac{1}{h_{m} \overline{\rho_{0} C_{p}}}\left[\frac{4 \pi}{9} \gamma \rho_{0}^{2} G R^{2}-\left(\rho_{F e} f_{0} L_{F e}+\rho_{S i}\left(1-f_{0}\right) L_{S i}\right)\right] .
$$

Here, $G$ is the gravitational constant, $\rho_{0}$ is the density of the undifferentiated 
planetary material, $R$ is the radius of the impacted planet. $L_{F e}$ and $L_{S i}$ are the latent heat required for melting respectively the metallic and the silicate phases. $f_{0}$ is the volume fraction of metal in the impacted planet. From equations (3), (4), and (5), $h_{m}$ represents the amount of heat that is used to increase the temperature inside and outside the isobaric core relative to the amount of heat used to increase the temperature by $\Delta T_{0}$ within the core and (Monteux et al., 2007)

$$
h_{m}=1+\frac{3(2 m-5)}{2(m-3)(m-2)} \approx 2.7
$$

135

136

Because $\Delta T_{0}$ depends on $\gamma$, the value for $\gamma$ requires some discussion. O'Keefe and Ahrens (1977) show that $\gamma$ depends on the impact velocity and that the fraction of energy converted into heat increases with impact velocity. Our assumption that $v_{i m p}=v_{e s c}$ implies that our modelled impactors are in a "slow impactor regime". From the measurements of O'Keefe and Ahrens (1977) a reasonable value for $\gamma$ is 0.3 . We apply this value in our calculations and discuss the influence of the uncertainty of this choice in section 5. An additional issue that requires mention is the assumption that there is no melting in the spherical shell around the isobaric core. For $\gamma=0.3$, calculation of $\Delta T_{0}$ as a function of planet radius shows that this approximation is strictly appropriate for planetary radii less than about $4000-4500 \mathrm{~km}$. For larger planets, latent heat may be consumed outside the isobaric core and so $\Delta T_{0}$ calculated with equation 5 may be overestimated. The magnitude of the overestimate is, however, unclear because the effect of melting on the presence and structure of the spherical shell region bounding the isobaric core is unknown. Nevertheless, assuming that the geometry of the region is similar to the case without melting, calculations show that the uncertainty in the value for $\gamma$ will have a greater influence on our 
estimates for $\Delta T_{0}$.

The magnitude of the temperature increase in Eq.5 depends on the two terms in square brackets: the left-hand term describes the kinetic energy released to heat the mantle on impact and the right-hand term is the latent heat consumed to melt the silicate and iron components of the mantle. For iron-silicate segregation to occur in the heated volume, $\Delta T_{0}$ must be positive and a sketch of the thermal state following impact is shown in Fig. $1\left(t_{2}\right)$. Thus, equating the two terms in parentheses in Eq.5 gives a critical impacted planet radius above which segregation is possible:

$$
R_{\text {crit }}>\left[\frac{\left.\rho_{F e} f_{0} L_{F e}+\rho_{S i}\left(1-f_{0}\right) L_{S i}\right)}{4 \pi / 9 \gamma \rho_{0}^{2} G}\right]^{1 / 2}
$$

For parameter values listed in Tab.1, $R_{\text {crit }}=1620 \mathrm{~km}$. Hence, impact heating on a Moon to a Mars-sized body will result in the separation of the dense iron phase from the silicate phase as a metallic diapir (Fig. 1). We note that whereas our assumption that $v_{i m p}=v_{\text {esc }}$ is appropriate for most planets, for moons this simplification underestimates the impact velocity, which will be governed by the gravitational potential of the central body and leads to a lower bound on $\Delta T_{0}$.

This temperature increase is superimposed on the pre-impact thermal regime of the protoplanet. The background thermal state depends on the accretionary process, which can lead to a temperature profile that increases with radius towards the surface (Senshu et al., 2002), and on the strong early rate of radiogenic heating, which augments the mean internal temperature of the planet (Yoshino et al., 2003). For simplicity we assume that $T_{0}$ is uniform and close to the eutectic temperature of the Fe-FeS system at 1 bar (Fei et al., 1997) and that the impacted body before the impact is a homogenous mixture of silicate and metal. 
For a given initial bulk composition, the radius of the diapir separated after a large impact $R_{F e}$ is a function of the impactor size $R_{i m p}$ and $f_{0}$ in the impacted planet and scales as $R_{F e}=\left(3 f_{0}\right)^{1 / 3} R_{i m p}$ (Monteux et al., 2009). The metal diapir sinks towards the centre of the planet over time-scales from several kyr to several Myr depending on the rheology of the protoplanetary mantle. Once at the center of the undifferentiated planet, this "metallic diapir" will be referred to as a "protocore".

\subsection{Heat partitioning during the sinking of the diapir}

The initial thermal state of the protocore is governed by its volume and the rate and location of viscous dissipation during its sinking, which depends on the viscosity ratio $\lambda$ (lower than 1) between the hot protocore and the relatively cold undifferentiated surrounding mantle (Ke and Solomatov, 2009; Samuel et al., 2010). For a given $\lambda$ the rate of viscous dissipation depends on the shear strain rate $\dot{\varepsilon}^{2}$, where $\dot{\varepsilon} \sim V_{S} / R_{F e}$ and $V_{S}$ is the sinking velocity. Monteux et al. (2009) showed that for small diapirs (i.e., $R_{F e}<70 \mathrm{~km}$ ), the rate of viscous dissipation is negligible and the metallic phase only cools during its sinking. In contrast, for $R_{F e}>70 \mathrm{~km}$ and for mantle and diapir viscosities that are equal (uniform viscosity), viscous deformation within the diapir and the surrounding mantle leads to dissipation that can increase the temperature of the metal diapir over its initial value from $T_{0}$ to $T_{\text {core }}>T_{0}+\Delta T_{0}$.

Fig. 1 (top left panel) shows where the viscous heating occurs when a spherical metallic diapir sinks within a Rybczynski-Hadamard analytical velocity field (Rybczynski, 1911; Hadamard, 1911) for 2 different viscosity ratios $\lambda$. For a uniform viscosity $(\lambda=1)$, viscous coupling between the metallic diapir and the surrounding material occurs and a fraction of gravitational energy is converted to heat up the diapir. However, for small viscosity ratios $\left(\lambda<\bigcirc\left(10^{-2}\right)\right)$, vis- 
cous heating is restricted to the surrounding mantle and is concentrated at the diapir's poles where strain rates are greatest (Fig. 1, top left panel and (Samuel et al., 2010)). In that case, the initial temperature of the protocore is lower than in the isoviscous case and remains close to the initial temperature of the diapir (i.e. $T_{\text {core }}=T_{0}+\Delta T_{0}$ ).

\subsection{Dynamical model for the sinking of an iron diapir}

\subsubsection{Physical model}

To investigate the dynamics of the metallic diapir separated after a large impact, we adapt the numerical finite volume model in spherical axisymmetric geometry of Monteux et al. (2009). In particular, neglecting the effects of adiabatic compression, conservation of energy applied to an undifferentiated planet of radius $R$ leads to

$$
\frac{D T}{D t}=\frac{\nabla^{2} T}{R a_{\chi}}+D_{\chi} \frac{\eta}{\eta_{0}} \phi
$$

where $T$ and $t$ are dimensionless temperature and time ( $T$ and $t$ are respectively normalized by $\Delta T_{0}$ and by a Stokes time $\left.t_{\text {Stokes }}=\eta_{0} / \Delta \rho g_{0} R\right) . R a_{\chi}=\frac{\Delta \rho_{0} g_{0} R^{3}}{\kappa \eta_{0}}$ is the compositional Rayleigh number and $D_{\chi}=\frac{\Delta \rho_{0} g_{0} R}{\overline{\rho_{0} C_{p}} \Delta T_{0}}$ is the dissipation number (with $\kappa$ the heat diffusivity and $\overline{\rho_{0} C_{p}}$ the average specific heat of the impacted body). $\Delta \rho_{0}$ is the density difference between metal and silicates. $g_{0}$ is the gravity at the surface of the impacted protoplanet. $\Delta T_{0}$ is the initial impact induced temperature increase below the impact site. $\phi$ is the dimensionless dissipation function and expresses the conversion of potential energy into heat:

$$
\phi=\tau: \nabla \mathbf{v}
$$

where $\tau$ is the dimensionless deviatoric stress tensor and $\mathbf{v}$ is the non-dimensional velocity (normalized with $v_{\text {Stokes }}=R / t_{\text {Stokes }}$ ). Prior to impact, we assume a homogenous temperature $T_{0}$ of the growing planet. The viscosity is $\eta=\eta_{0} \lambda^{T}$ 
Assuming the protoplanet is incompressible, the other dimensionless governing equations are continuity

$$
\boldsymbol{\nabla} \cdot \mathbf{v}=0
$$

and momentum conservation assuming infinite Prandtl number

$$
-\nabla P+\nabla \cdot\left(\frac{\eta}{\eta_{0}}\left[\nabla \mathbf{v}+[\nabla \mathbf{v}]^{T}\right]\right)+\left(\frac{T}{B}-f\right) r \mathbf{e}_{\mathbf{r}}=0,
$$

where $P$ and $r$ are the non-dimensional pressure and radius $(P$ and $r$ are respectively normalized by $\eta_{0} / t_{\text {Stokes }}$ and $R$ ). $\mathbf{e}_{\mathbf{r}}$ is the radial unit vector and $f$ represents the volume fraction of metal. Following Monteux et al. (2009), we introduce the buoyancy ratio $B=\Delta \rho_{0} /\left(\rho_{0} \alpha \Delta T_{0}\right)$ where $\rho_{0}$ and $\alpha$ are the density and thermal expansion of the undifferentiated material (see Tab.1 for values). Gravity depends on the radial position $r$ and $g(r)=\frac{4}{3} G \pi \rho_{0} r=g_{0} \frac{r}{R}$.

After an impact and the subsequent differentiation, the metallic phase and the purely silicate phase are separated within the thermal anomaly (Fig.1, $t_{3}$ ). Hence a chemical readjustment occurs leading to the sinking of the dense iron though the lighter undifferentiated material. The buoyancy force that drives the flow of the diapir towards the centre of the protoplanet increases with the volume fraction of metal $f$. In our models, we consider only two chemical phases meaning that $f$ varies between 1 (pure metal) and 0.17 (undifferentiated material with chondritic composition). During sinking, chemical diffusion is 
negligible. The metal volume fraction $f$ is then simply advected by the flow :

$$
\frac{\partial f}{\partial t}+v \cdot \nabla f=0 .
$$

234

\subsubsection{Simplifications of the governing equations}

We make a number of approximations to simplify the analysis of the initial thermal state of the protocore. We neglect the thermal buoyancy $T / B$ in the momentum equation as the buoyancy number $B$ is very large (the density difference between metal and silicates is 140 to 560 times larger than the thermal density variations). In addition, in treating the metal-silicate separation, our model neglects multiphase dynamics associated with core-mantle segregation (Golabek et al., 2008; Srámek et al., 2010).

Prior to the impact, the planet is undifferentiated meaning that the impact and the subsequent local differentiation leads to the first episode of core formation. Moreover, as this study focuses on the thermal state of the metallic phase once at the centre of the planet, we do not consider the dynamical effects of the purely silicate phase that spreads beneath the impacted planet surface while the diapir sinks to the centre of the planet (Reese and Solomatov, 2006; Monteux et al., 2009).

\subsubsection{Numerical model}

We implement a finite volume numerical model to solve Eq.8, Eq.10, Eq.11 and Eq.12 in axisymmetric spherical geometry in a $200 \times 400$ grid. We use a stream function formulation for the equations of motion with a direct implicit inversion method (Schubert et al., 2001). Eq.8 and Eq.12 are solved by an Alternating Direction Implicit (ADI) scheme (Peaceman and Rachford, 1955; Douglas, 1955). The stream function, temperature and compositional fields 
249

250

are described by a second-order approximation in space. To limit numerical diffusion when solving the transport equations, especially for the compositional field, we use a Total Variation Diminishing Superbee scheme (Roe, 1986; Laney, 1998) implemented in an implicit way (Srámek et al., 2010) which enables high resolution of purely advective fields. Mechanical boundary conditions are freeslip at the surface and along the symmetry axis. Thermal boundary conditions are isothermal at the surface and insulating along the symmetry axis.

\section{Magnetic field considerations}

\subsection{Temperature of the protocore}

Once at the centre of the planet, core cooling depends on the temperature of the protocore and the thermal state and melting temperature of the silicate at the protocore-mantle boundary. As discussed above, the temperature of the protocore depends on the post-impact temperature difference $\Delta T_{0}$ (cf. Eq.5). The maximal temperature increase after sinking is (Monteux et al., 2009) is :

$$
\Delta T \approx \Delta T_{0}\left(1+a(\lambda)\left(\frac{r_{0}}{R}\right)^{2} \frac{\Delta \Theta}{\Delta T_{0}}\right)
$$

where $a(\lambda)$ is the fractional amount of viscous dissipation in the metal during the sinking of the iron towards the centre of the planet. $r_{0}$ is the initial distance between the inertial centre of the metallic diapir (noted i.c. in (Fig. 1, $\left.\boldsymbol{t}_{\mathbf{3}}\right)$ ) and the centre of the planet before the diapir starts to sink. $\Delta \Theta$ is the maximum temperature increase in the unrealistic case in which all the potential energy released by the diapir is used to heat up the metal phase. Assuming the impact velocity is the escape velocity of the planet (cf., section 2) then $\Delta \Theta / \Delta T_{0}$ is constant, independent of the planet size and equal to 11.8 (Monteux et al., 
2009) and the temperature of the metallic phase at the end of the sinking is

$$
T_{\text {core }} \approx T_{0}+\Delta T=T_{0}+\Delta T_{0}\left(1+a(\lambda)\left(\frac{r_{0}}{R}\right)^{2} \frac{\Delta \Theta}{\Delta T_{0}}\right) .
$$

During the sinking of the metallic diapir, its pressure increases and leads to additional heating by compressional effects. This heating process is inversely proportional to the buoyancy ratio $B$ (Monteux et al., 2009). In our models, $B$ is large enough to consider that this thermal effect is negligible compared to viscous heating (Samuel and Tackley, 2008).

\subsection{Conditions for an early magnetic field.}

Three conditions are required to drive a dynamo on a growing planet:

1. The heat flow $Q$ out of the core must exceed the adiabatic value $Q_{A}$ such that convection can occur (Stevenson et al., 1983). This condition is

$$
Q>Q_{A}=\frac{k_{F e} \alpha_{F e} g_{c} T_{F e}}{C_{p, F e}} 4 \pi R_{F e}^{2}
$$

where $k_{F e}, \alpha_{F e}, T_{F e}$ and $C_{p, F e}$ are respectively the thermal conductivity, the thermal expansion, the temperature and the heat capacity of the metallic phase and $g_{c}$ is the gravity at the surface of the protocore (see Tab.1 for values). In our model, once the metallic phase has reached the center of the impacted planet, $T_{F e}$ decreases monotonically with time due to heat conduction to the surrounding mantle.

2. The ratio of the rate at which gravitational potential energy is released by convection to the rate of ohmic dissipation, $\Phi$, must exceed a critical value (Buffett, 2002): 


$$
\frac{Q}{\Phi}>\frac{1}{\epsilon_{T}}
$$

Here, $\epsilon_{T}$ is the Carnot-style efficiency for thermal convection. We note that a dynamo can be generated even with a subadiabatic heat flow if the compositional convection is important (Buffett, 2002; Christensen and Wicht, 2008). Here, we do not consider the effect of chemical convection or the presence of an inner core. Assuming that the characteristic length scale of the flow leading to magnetic field generation is the radius of the protocore, $\Phi$ can be approximated as (Buffett, 2002):

$$
\Phi=\left(\frac{\nu \bar{B}^{2}}{\mu}\right) \frac{4}{3} \pi R_{F e}
$$

where $\bar{B}$ is the average strength of the magnetic field within the core, $\nu$ is the magnetic diffusivity and $\mu$ is the magnetic permeability (see Tab.1 for values). In the absence of constraints on $\bar{B}$ for early planets, we assume a current Earth-like value of $2.5 \mathrm{mT}$ (Kuang and Bloxham, 1997) that is independent of the protocore size or the planetary radius. The efficiency of thermal convection is given by (Buffett, 2002)

$$
\epsilon_{T}=\frac{0.8 \pi}{3} \frac{\alpha_{F e} G \rho_{F e} R_{F e}^{2}}{C_{p, F e}}\left(1-\frac{Q_{A}}{Q}\right)
$$
with $\rho_{F e}$ the density of the metallic phase and $G$ the gravitational constant.

3. The structure of the convective motions carrying magnetic field lines must be sufficiently complicated to favor self-sustaining dynamo action. A measure of this complexity is that the magnetic Reynolds number (Christensen 
and Aubert, 2006)

$$
R e_{m}=\frac{U L}{\nu}>R e_{m}^{c r i t}=O\left(10-10^{2}\right)
$$

Here, $L$ and $U$ are the characteristic length and velocity scales for the flow within the protocore and $\nu$ is the magnetic diffusivity of the metal phase. Whereas the natural length scale in the problem is the depth of the convecting iron layer, the choice of an appropriate velocity scale depends on the leading order force balance (Christensen, 2010). As the rotation rate of growing planets is potentially time-dependent and poorly constrained a convenient and reasonable choice is based on a balance between inertial and buoyancy forces and is (Stevenson, 2003a):

$$
U \sim\left(\frac{q \alpha_{F e} g_{c} R_{F e}}{\rho_{F e} C_{p, F e}}\right)^{1 / 3}
$$

where $\mathrm{q}$ is the heat flux out of the core $\left(q=Q /\left(4 \pi R_{F e}^{2}\right)\right)$. Taking $L=$ $R_{F e}, g_{c}=4 / 3 \pi G \rho_{F e} R_{F e}$, the combination of Eq.19 and Eq.20 leads to the condition

$$
R e_{m}=\left(\frac{4 \pi G q \alpha_{F e} R_{F e}^{2}}{3 C_{p, F e}}\right)^{1 / 3} \frac{R_{F e}}{\nu}>O\left(10-10^{2}\right)
$$

Among the three criteria above, the first is typically considered a necessary condition for a thermally-driven dynamo. (Subadiabatic dynamos are possible if e.g., compositional gradients also drive convection.). As we shall see in Section 4 , this condition $\left(Q>Q_{A}\right)$ is easily met in our models. However it is not a sufficient condition for dynamo action, as indicated by the other two conditions. The second condition assumes an Earth-like dynamo: in particular it assumes an Earth-like value for the root mean square magnetic field in the 
core, a particular efficiency for conversion of gravitational potential energy into magnetic energy and a particular toroidal-to-poloidal field conversion efficiency. The third condition is a more general criterion for dynamo action, and whereas scalings other than Eq.20 for the velocity field are possible, we have chosen one that does not depend upon knowing the early rotation rate of the planet or moon.

\subsection{Heat flow across the protocore: Analytic considerations}

The three conditions required for an early dynamo (Eq.15, Eq.16 and Eq.21) all impose conditions on the heat flow out of the protocore. At the protocoremantle boundary there are two asymptotic heat transfer regimes. The first is a conductive transfer across a thermal boundary layer growing on the mantle side where the corresponding heat flow $Q_{\text {cond }}$ is :

$$
Q_{\text {cond }} \sim \frac{4 \pi k \Delta T R_{F e}^{2}}{\delta}
$$

where $\delta \approx 2 \sqrt{\kappa t}$ is the thickness of the conductive boundary layer at any time $t$. As $t$ becomes large $\delta=c_{2} R_{F e}$ (where $c_{2}$ is an order 1 constant (Monteux et al., 2009)). $k$ is the thermal conductivity of the surrounding material. For computational reasons, in our numerical models, we make the approximation that thermal conductivities for metallic and undifferentiated material are the same (i.e., $k=k_{F e}=k_{S i}$, Table 1 ). In reality, the thermal conductivity of the core material is $\approx 10$ times bigger than the conductivity for the silicates. Hence, this simplification underestimates the protocore heat flow and our criteria for dynamo action is more restrictive than in the more realistic case of unequal silicate and metal thermal conductivities.

As illustrated in Fig.2, the temperature of the protocore might overcome 
the solidus temperature of the surrounding material depending depending on its chemical composition and on the impacted planetary radius. Hence, a melt layer may form above the protocore (see also section 5.2). As this melt layer grows in thickness (and assuming it is greater than a few tens of meters thick), vigorous convection will enhance core cooling over the conductive case and lead to a heat flow that is independent of the thickness of the melt layer and of the form (Turner, 1973):

$$
Q_{\text {conv }} \sim 0.8 \pi R_{F e}^{2}\left(\frac{\rho_{S i}^{2} g_{c} \alpha_{S i} k^{2} C_{p, S i}}{\mu_{\text {melt }}}\right)^{1 / 3}\left(T_{\text {core }}-T_{\text {melt }, S i}\right)^{4 / 3}
$$

where $\rho_{S i}, \alpha_{S i}, C_{p, S i}$ and $\mu_{m e l t}$ are respectively the density, the thermal expansion, the heat capacity and the viscosity of the molten silicate layer (see Tab.1 for typical values). Eventually, the heat flux out of the core will, of course, become balanced by the heat flux across the overlying solid mantle and the melt layer will have a quasi-steady thickness.

\section{Numerical results}

\subsection{Diapir descent: The initial protocore temperature}

In our models, where we specify the viscosity of the impacted planet as $\eta_{0}=10^{22}$ Pas, metallic diapirs descend to the centre of the planet relatively quickly (e.g. in less than $10 \mathrm{Myr}$ for $R_{F e}=400 \mathrm{~km}$ ) (Monteux et al., 2009). During the sinking of the diapir the temperature difference between the protocore and the mantle increases rapidly in response to viscous heating depending on the fraction of gravitational energy converted into heat $(a(\lambda))$. Then this temperature difference decreases as the diapir cools once it reaches the centre of the planet. 
We monitor $T_{\text {core }}$ for diapirs in the range of $150-500 \mathrm{~km}$ and planet radii in the range of $2000-4000 \mathrm{~km}$. For the diapir radii range studied here, the protocore temperature at the end of sinking is given by Eq.13 and Eq.14. The results from our numerical models for uniform viscosity contrasts are shown in Fig. 2 and are in agreement with Eq.14. Theoretical predictions of $T_{\text {core }}$ are represented in Fig. 2 with and without taking into account the effect of melting in the isobaric core after impact (grey and black solid lines respectively). The region in between defines the range of initial temperature and the difference between these two theoretical models is within the range of uncertainties of $\gamma$. For a uniform viscosity, viscous coupling between metal and undifferentiated material is important and $a(\lambda) \approx 15 \%$ (Monteux et al., 2009). As the viscosity contrast between the hot metallic phase and the surrounding mantle increases, the fraction of gravitational potential energy converted into heat in the metallic phase decreases (see section 2.2). That is, as the mantle/core viscosity ratio becomes very large $a(\lambda) \rightarrow 0$ and viscous dissipation is concentrated in the mantle (Monteux et al., 2009). In this limit, the initial temperature of the protocore after the sinking is $T_{\text {core }}=T_{0}+\Delta T_{0}$. The predictions of Eq.14 for this asymptotic case are shown in Fig. 2 (dashed line). As expected, the reduced dissipation in the metallic diapir leads to a lower predicted $T_{\text {core }}$.

A high temperature contrast between the protocore and the surrounding undifferentiated mantle can eventually lead to the formation of a molten layer above the protocore. In Fig. 2, we include the chondrite solidus temperature from Agee (1997) and the dry peridotite solidus from Zerr and Boehler (1994) at the centre of the impacted planet as a function of the planetary radius. This effect will be more precisely discussed in section 5.2. 
367

Because of the practical difficulty in obtaining accurate solutions for very large viscosity variations, we restrict our numerical studies to the isoviscous case, which is well-explained by our theory. The agreement between our isoviscous theory and the numerical solutions give us confidence that application of the theory for the variable viscosity case is justified. From Figure 2 these isoviscous solutions will overestimate the core temperature by $\approx 10-40 \%$, depending on the protoplanetary radius. Thus, quantitatively, the uncertainties of this enhanced heat flow for predicting dynamo action are not so large. From Eq.13, assuming $a(\lambda)=15 \%$, the temperature difference between the protocore and the cold surrounding material in the isoviscous model is overestimated by a factor $\approx 2.8$. From Eq.21 $R e_{m} \propto Q^{1 / 3} \propto \Delta T^{1 / 3}$ and, thus, the magnetic Reynolds number will be a factor of about 1.4 too large (i.e., it is still accurate to $O(1)$ ).

\subsection{Dynamo initiation in a conductive heat transfer case}

To determine whether or not a dynamo is initiated in the protocore, we monitor the average heat flow $Q$ across the protocore-mantle boundary as a function of time for a wide range of $R-R_{F e}$ conditions. We stop calculations $50 \mathrm{Myr}$ after the impact because at this time the core is probably fully formed and because of the potential onset of mantle convection, the thermal consequences of which are beyond the scope of this paper (Behounkova and Choblet, 2009).

$R-R_{F e}$ conditions studied, we find that $Q>>Q_{A}$ after impact, which means that core convection can occur (see Eq.15). For example, once a metallic diapir with $R_{F e}=300 \mathrm{~km}$ has reached the centre of a planet with radius $R=3500 \mathrm{~km}$, it starts to cool and over the 50 Myr period studied, $Q / Q_{A}$ decreases from 32.5 to 20 . For $R=2000 \mathrm{~km}, Q / Q_{A}$ decreases from 15 to 10 over the same time interval (in the calculation of $Q_{A}$ we consider that $k_{F e}=40 \mathrm{~W}$ $\left.\mathrm{m}^{-1} \mathrm{~K}^{-1}\right)$. Assuming that the thickness of the thermal boundary layer around 
In the special case of the impacted planet being Earth-like from a magnetic perspective, convection within the protocore has to be sufficiently vigorous to supply energy at a rate that compensates for the loss due to ohmic decay. We monitor the ratio of the heat flow to the ohmic dissipation as a function of time. Assuming a conductive heat transfer across the protocore mantle boundary, $k=k_{S i}$ and $\delta \sim R_{F e}$, from Eq.13, Eq.17 and Eq.22 we obtain

$$
\left(\frac{Q_{\text {cond }}}{\Phi}\right) \sim \frac{3 \mu k \Delta T_{0}}{\nu \bar{B}^{2}}\left(1+a(\lambda) \frac{\Delta \Theta}{\Delta T_{0}}\right) .
$$

the protocore scales with the size of the protocore, this corresponds to a temperature difference of $\approx 300$ to $2700 \mathrm{~K}$ between the metallic phase and the overlying mantle material (see Fig. 2). However, as discussed in Section 3, this condition alone does not guarantee dynamo action, and so we next examine the additional requirements for a dynamo.

which is a conservative bound on whether a dynamo will occur (we consider here that $\left.r_{0} / R \approx 1\right) . a(\lambda)$ and $\Delta \Theta / \Delta T_{0}$ are independent of $R$. However, from Eq.5, $\Delta T_{0} \propto R^{2}$. Hence, $\left(Q_{\text {cond }} / \Phi\right)$ varies with $R^{2}$. Figure 3 shows $Q / \Phi$ as a function of the planet radius for different iron diapir sizes and different times (1 Myr and $50 \mathrm{Myr}$ ), after the diapir reaches the centre of the impacted planet. The dashed line is the theoretical prediction from Eq.24 for a uniform viscosity case (i.e., $a(\lambda)=15 \%$ ). Numerical results are consistent with Eq.24 at very early time (just after the metallic diapir has reached the centre of the planet). At larger time, the protocore cools down and $Q / \Phi$ decreases. The temperature of the metallic phase after sinking and hence the initial heat flow across the protocore is a sensitive function of the viscosity contrast between the metallic phase and the surrounding mantle (see section 3.2). The theoretical value of the initial conductive heat flow when the viscosity of the hot metallic fraction is 
much smaller than the viscosity of the relatively cold undifferentiated material is given by Eq.13 and Eq.22 with $a(\lambda)=0 \%$. The black dotted line in Fig.3 shows the corresponding theoretical prediction for $\left(Q_{c o n d} / \Phi\right)$ immediately after sinking as a function of $R$ for $a(\lambda)=0 \%$. In this case, the theoretical value for $\left(Q_{\text {cond }} / \Phi\right)$ is different by a factor of 2.8 compared with the uniform viscosity case, consistent with expectations from Rybczynski-Hadamard effects.

In all sets of planetary and metallic diapir radii studied here $Q>>Q_{A}$. Consequently, the condition for the presence of a dynamo from the combination of Eq.16 and Eq.18 simplifies to:

$$
\frac{Q}{\Phi}>\frac{3}{0.8 \pi} \frac{C_{p, F e}}{G \alpha_{F e} \rho_{F e} R_{F e}^{2}}
$$

Figure 4 shows $Q / \Phi$ as a function of the protocore radius resulting from a single impact for different planet sizes in uniform viscosity cases. For $t=1$ Myr after the end of the sinking (black filled symbols), and for a given planetary radius, different trends appear as a function of $R_{F e}$. The ratio $Q / \Phi$ is initially larger for larger bodies because $Q \propto \Delta T_{o} \propto R^{2}$. However, as mentioned in section 2.2 when $R_{F e}<70 \mathrm{~km}$, no viscous heating occurs during the sinking (Monteux et al., 2009) as cooling by diffusion is faster than viscous heating. As $R_{F e}$ increases from $70 \mathrm{~km}$ radius, viscous heating becomes more and more efficient and leads to an increase of the initial heat flux and as a consequence of $Q / \Phi$. When $R_{F e}$ reaches a critical value $(\approx 300 \mathrm{~km})$, the simplification $r_{0} / R=1$ is less and less valid since the diapir is initially buried deeper. Hence, for a given $R$, as $R_{F e}$ increases $r_{0} / R$ decreases so the initial temperature of the protocore, the heat flux and $Q / \Phi$ also decrease. With subsequent cooling, the reduction in core temperature over a given time period $\Delta t$, say, scales as $Q \Delta t /\left(\overline{\rho C_{p}} R_{F e}^{3}\right)$. Because heat flow will decline with the core-mantle temperature difference, and 
441

the incremental change in core temperature $\propto\left(1 / R_{F e}^{3}\right)$, small protocores cool faster than big ones. Hence, for a given $R$, and for $t=50 \mathrm{Myr}$ after the end of the sinking (white filled symbols), $Q / \Phi$ increases as a function of $R_{F e}$.

In Fig.4, the black dashed line represents the condition required to get a dynamo from Eq.25. Results show that a dynamo is difficult to initiate with a small volume of metallic phase (i.e. small impactors). Once the protocore radius is larger than $400 \mathrm{~km}$ (i.e. $R_{i m p}>500 \mathrm{~km}$ ), however, an early dynamo can be initiated in the first $50 \mathrm{Myr}$ in a planet with a radius greater than $2500 \mathrm{~km}$ and in the absence of a molten layer surrounding the protocore. As the planet size increases, the initial protocore temperature increases and the duration of the early dynamo is longer (over $50 \mathrm{Myr}$ ). As the protocore size increases, its characteristic cooling time increases and the duration of the early dynamo is also longer. However, a large viscosity contrast between hot iron and cold undifferentiated material will decrease the initial heat flow by a factor of 2.8 (see Fig. 3), and increase the dynamo threshold from Eq.25. The dotted line in Fig.4 illustrates this theoretical effect. Fig.4 shows that a dynamo can still be initiated after an impact, but an increase of the impactor and impacted planet radii are needed relative to the isoviscous case. Hence an impact with $R_{i m p}>625$ km (i.e. $R_{F e}>500 \mathrm{~km}$ ) on a Mars size body (i.e. $R \sim 3500 \mathrm{~km}$ ) can initiate an early magnetic field. The duration of this early dynamo increases with the size of the impactor.

Dynamo generation requires a magnetic Reynolds number larger than a critical value (see Eq.21). We monitored the mean heat flux $q$ as a function of time and calculated the corresponding magnetic Reynolds numbers. Fig.5 shows the results for the two extreme planetary radii (i.e. $R=2000 \mathrm{~km}$ and $R=4000 \mathrm{~km}$ ) 
462

and the range of protocore sizes studied here. The two lines (solid and dashed) correspond to $q=q_{A}=Q_{A} /\left(4 \pi R^{2}\right)$ with $R=2000 \mathrm{~km}$ and $R=4000 \mathrm{~km}$ respectively. Three regimes emerge. Below, the solid line $q<q_{A}$ and no convection occurs. Above the solid line and below $R e_{m}^{\text {crit }}$, convection occurs but no dynamo is generated. Above these criteria, the thermally-driven convective flow within the protocore can generate a dynamo. As mentioned above, the magnetic Reynolds number will be a factor of about 1.4 too large in the isoviscous compared with the temperature-dependent viscosity case. The dotted line represents the values for $R e_{m}$ expected from the temperature-dependent viscosity model (i.e. $R e_{m}$ from isoviscous models divided by a factor 1.4). This figure illustrates three results. First, temperature-dependent viscosity has a weak influence on the $R e_{m}$ values obtained in this study. Second, as the heat flux out of the protocore obtained from our numerical models is $\sim 20$ larger than the adiabatic heat flux $q_{A}$ (see Eq.15 and discussion above), then $\operatorname{Re}_{m}(q)>>\operatorname{Re}_{m}\left(q_{A}\right)$. Third, $R e_{m}$ easily exceeds the critical value needed to generate a dynamo in the whole range of planet and protocore radii studied here.

\section{Discussion}

These results provide an important link between the accretion and differentiation histories of the terrestrial planets and their potential for early magnetic fields. They suggest that the presence or absence of an early magnetic field on a planet could be related to its accretionary history.

\subsection{Application to Solar System objects}

Our results indicate that for bodies in the range $R=2000-4000 \mathrm{~km}$ and protocore radii in the range $300-650 \mathrm{~km}$, the core heat flow exceeds the adiabatic heat flow, and $R e_{m}$ easily exceeds $R e_{m}^{c r i t}$ for $50 \mathrm{Myr}$ after the iron diapir reaches 
473 protocore-mantle boundary. These conditions suggest that for the range of $R$

474 $R_{F e}$ conditions studied, dynamo action is possible. A more restrictive criterion

475 is that the heat flow must also exceed the ohmic dissipation under Earth-like

the center of the planet and in the absence of additional mantle melting at the conditions. In this case, and assuming no mantle melting, we find that a large impact (a $625 \mathrm{~km}$ radius impactor, or $R_{F e}=500 \mathrm{~km}$ ) onto a Mars-size (i.e., $R$ $=3500 \mathrm{~km}$ or greater) undifferentiated planet is needed to initiate a dynamo that would persist at least for $\sim 50$ Myr (Fig. 4).

As noted earlier the ohmic dissipation criterion has several underlying assumptions about the dynamo action. In particular, we note that the Earth-like value of the magnetic field in the core $\bar{B}$ may not be applicable to other bodies. For example, Mercury, the only other inner solar system body to have a presentday dynamo, has a magnetic field at its surface that is 100 times weaker than Earth's surface field. While the origin of this weak field is not well understood, it is at least reasonable to propose that Mercury's core field could be weaker than Earth's. A factor of two decrease in the core field results in a factor of four increase in $Q / \Phi$, allowing dynamo action for smaller impactors and impacted bodies.

Independently of its mode of formation, such a scenario is more difficult to envision for the Moon for two reasons. First, the Moon is smaller $(R=1700$ $\mathrm{km}$ ), and in our models, the heat flow across the core mantle boundary does not reach the critical value required to overcome ohmic dissipation. Second, its volumetric metallic fraction is only $1-2 \%$ meaning that the impactor size needed to differentiate a large volume of metal is unrealistically large. However, our models do not consider any contribution of the central body to the impact 


\subsection{The effect of a molten lower mantle layer on dynamo duration}

If the rocks at the protocore-mantle boundary are at sufficiently high temperature and pressure to be close to their melting temperature, heat transfer from the protocore can cause the progressive growth of a mantle melt layer (see Eq.5 and Fig.2). Assuming that the undifferentiated mantle surrounding the protocore is homogenous, that gravity decreases linearly with depth and that $\left(R_{F e} / R\right)^{2}<<1$, the pressure at the core mantle boundary is:

$$
P_{C M B} \approx \frac{2}{3} \pi G \rho_{0}^{2} R^{2}
$$

514 For the impacted planet radii range in our models, $P_{C M B}$ ranges from 10 to 50 515 GPa. The formation of a molten layer at the top of the core depends on the 516 initial temperature of the protocore which strongly depends on the impacted 
planet radius $R$ (see Eq.5). Melting occurs when the initial protocore temperature is larger than the solidus of the surrounding material. In the isoviscous case, Fig. 2 shows that the protocore temperature overcome the solidus temperature of the surrounding mantle only when $R>3500 \mathrm{~km}$ with a chondritic composition (Agee, 1997) or when $R>4500 \mathrm{~km}$ with a dry peridotite composition (Zerr and Boehler, 1994). When the viscosity of the diapir is smaller than the surrounding undifferentiated solid mantle, the initial temperature of the protocore is smaller and it is more difficult to form a molten layer unless the pre-impact temperature $T_{0}$ at the centre is increased by $\approx 500 \mathrm{~K}$. As mentioned in section 2.1, the pre-impact temperature is a function of the accretion rate and of the early radiogenic heating. In our models the pre-impact thermal state is homogenous and $T_{0}=1250 \mathrm{~K}$. Hence, in our single impact scenario, the formation of a molten layer at the top of the protocore without additional silicate differentiation would require a pre-impact temperature that increases with depth.

The conductive model and condition given by Eq.25 is not appropriate if heat transfer from the core melts the lower mantle. Vigorous convection within a growing melt layer will enhance the heat transfer from the protocore. Thus, mantle melting can increase the likelihood of dynamo action over the conditions identified in Fig.4 but, in return, can decrease the duration of an early dynamo as heat will be removed more quickly. Hence, a key issue is to know whether convection in the melt layer will cause the core to freeze. In the case of a molten layer, the duration of a dynamo is limited by the time needed to remove the excess heat (the specific heat plus the latent heat of crystallization) from the 
protocore with a heat flow $Q$. In that case, the duration of the dynamo is :

$$
t_{\text {dynamo }} \approx \frac{4 \pi R_{F e}^{3} \rho_{F e}\left[C_{p, F e}\left(T_{\text {core }}-T_{m e l t, F e}\right)+L_{F e}\right]}{3 Q}
$$

where $T_{m e l t, F e}$ and $L_{F e}$ are the melting temperature and the latent heat of the metallic phase (see Tab.1 for typical values). Figure 6 shows the time needed to remove the heat excess as a function of the planetary radius for 3 different heat transfer types: solid state conduction, time-dependent conduction and convection within a molten layer. Our results show that this time can vary with several orders of magnitude. In the case of a conductive layer, the time to remove the heat excess is very long $\left(\approx 10^{9} \mathrm{yrs}\right)$ and the duration of an early dynamo is essentially governed by the time during which vigorous convection occurs within the protocore $\left(\approx 10^{6}-10^{7} \mathrm{yrs}\right)$. However, in the case where a molten convecting layer forms above the protocore, core freezing occurs in $10^{3}-10^{4} \mathrm{yrs}$, potentially limiting the duration of the dynamo to $<10$ kyr. As underlined in Fig..2, the extent of melting depends on the composition of the surrounding rocks and on the easiness to cross the solidus. Hence, the composition of the early mantle is also a key parameter in the duration of the early dynamo.

\subsection{Future investigations}

This dynamo initiation model is a first step toward a more general model of core formation. From the results of our models, several questions need to be addressed:

1. How do hyper-velocity impacts affect the heat repartition after an impact and how does this in turn enhance an impact-induced early dynamo? The fraction of kinetic energy $(\gamma)$ that is retained as heat below the impact site and the spatial heat repartition after an impact $\left(h_{m}\right)$ are key parameters to characterize the early inner thermal state of growing planets. Better 
constraints on these parameters would greatly assist any models that investigate the thermal consequences of impacts early in a planet's history.

2. How multiple diapirs from multiple impacts will affect the early protocore thermal state? The sinking of multiple metallic diapirs will strongly affect the thermal state of both the core and the mantle and may play a key role on the temperature contrast between these two reservoirs (Golabek et al., 2009; Samuel et al., 2010). This prospect is currently beyond the scope of this study because of the geometry used in our numerical models (axisymmetric spherical). However, our study gives a parametrization of the inner thermal consequences after a single large metallic diapir sinking. The scalings proposed here could be used to compute more accurate one dimensional thermal evolution models of growing planets.

3. What is the influence of the timescale between two successive impacts? If a second impact occurs shortly after the first (i.e., within one thermal diffusion timescale for first metallic diapir diameter), the thermal effect of the first impact will still be present within the mantle and the second diapir will sink into a warmer and less viscous mantle. The second diapir will descend more quickly. Moreover, the viscosity contrast between the second diapir and the hotter mantle will decrease and supplementary viscous heating will occur within the second metallic diapir (see section 2.2).

4. In the framework of a late giant impact between two differentiated bodies, what is the effect of the core merging on the dynamo activity? The thermal effect of giant impact on dynamos has been recently studied (Roberts et al., 2009; Reese and Solomatov, 2010) but the dynamics of merging between the preexisting core and the impactor's core and the thermal consequences 
have also to be considered in future models. Within the framework of our model, a giant impact might kill a preexisting dynamo depending on size of the impactor as the thermal effect of both the impact and the viscous heating during the sinking may reduce the total heat flow across the CMB.

5. How would a second large impact or merging phenomenon change the HfW systematics? Depending on the efficiency of equilibration between the impactor's core and the impacted body's mantle, this merging phenomenon may be a major feature of the current $\mathrm{W}$ isotopes composition of planets and influence the Hf-W chronology of accretion (Jacobsen, 2005).

\section{Conclusions}

The dynamics of terrestrial planetary accretion governs their differentiation histories and their potential for early magnetic fields. We have shown that under Earth-like conditions for ohmic dissipation in the core impactors (with $R_{i m p}>625 \mathrm{~km}$ ) leading to major differentiation events during early stages of planetary formations can initiate early dynamos. If the ohmic dissipation criterion is relaxed, allowing dynamo action simply if the critical magnetic Reynolds number in the core is exceeded, we find that dynamo action is possible for a larger range of impactors and planets (notably smaller impactors and smaller impacted bodies). Such early dynamos can persist for several kyr to several Myr depending on the size of both the impactor and the impacted body and depending on the heat transfer regime across the protocore. The duration of the early dynamo is also a strong function of the rheology of the early mantle of the impacted body. This rheology determine the sinking velocity of the metallic diapir, the amount of viscous heating during the sinking and the ease to remove the heat from the protocore in the case of a molten layer. 
610

611

612

\section{Acknowledgements}

The authors thank Laboratoire de Sciences de la Terre de Lyon for its computing time and R. Pawlowicz for useful discussions. We also thank three anonymous reviewers for thoughtful and constructive comments. This project was funded by a Lavoisier fellowship and the Canadian Institute for Advanced Research. M. Jellinek and C.L. Johnson ackowledge support from NSERC and C.L. Johnson from NASA's Planetary Geology and Geophysics Program.

\section{References}

Acuña, M., et al. (1999), Global distribution of crustal magnetization discovered by the mars global surveyor MAG/ER experiment, Science, 284, 790-793.

Agee, C. B. (1997), Melting temperatures of the Allende meteorite: implications for a Hadean magma ocean, Phys. Earth Planet. Int., 100, 41-47.

Behounkova, M., and G. Choblet (2009), Onset of convection in a basally heated spherical shell, application to planets, Phys. Earth Planet. Int., 176, 157-173.

Breuer, D., and T. Spohn (2003), Early plate tectonics versus single-plate tectonics on Mars: Evidence from magnetic field history and crust evolution, $J$. Geophys. Res. (Planets), 108, 5072-5085.

Buffett, B. A. (2002), Estimates of heat flow in the deep mantle based on the power requirements for the geodynamo, Geophys. Res. Lett., 29(12), 1-4.

Christensen, U. R. (2010), Dynamo Scaling Laws and Applications to the Planets, Space Sci. Rev., 152, 565-590. 
Christensen, U. R., and J. Aubert (2006), Scaling properties of convectiondriven dynamos in rotating spherical shells and application to planetary magnetic fields, Geophys. J. Int., 166, 97-114.

Christensen, U. R., and J. Wicht (2008), Models of magnetic field generation in partly stable planetary cores: Applications to Mercury and Saturn, Icarus, $196,16-34$.

Cisowski, S. M., D. W. Collinson, S. K. Runcorn, A. Stephenson, and M. Fuller (1983), A review of lunar paleointensity data and implications for the origin of lunar magnetism, J. Geophys. Res., 88, 691-704.

Croft, S. K. (1982), A first-order estimate of shock heating and vaporization in oceanic impacts, vol. 190, 143-152 pp., Geological Implications of Impacts of Large Asteroids and Comets on Earth, edited by T.L. Silver and P.H. Schultz, Spec. Pap. Geol. Soc. Am.

Douglas, J. (1955), On the numerical integration of $\frac{\partial^{2} u}{\partial x^{2}}+\frac{\partial^{2} u}{\partial y^{2}}=\frac{\partial u}{\partial t}$ by implicit methods, J. Soc. Ind. Appl. Math., 3, 42-65.

Elkins-Tanton, L. T., S. E. Zaranek, E. M. Parmentier, and P. C. Hess (2005), Early magnetic field and magmatic activity on Mars from magma ocean cumulate overturn, Earth Planet. Sci. Lett., 236, 1-12.

Fei, Y., C. M. Bertka, and L. W. Finger (1997), High-Pressure Iron-Sulfur Compound, Fe3S2, and Melting Relations in the FeFeS System, Science, 275, $1621-1623$.

Garrick-Bethell, I., B. P. Weiss, D. L. Shuster, and J. Buz (2009), Early Lunar Magnetism, Science, 323, 356-359.

Golabek, G. J., H. Schmeling, and P. J. Tackley (2008), Earth's core formation 
aided by flow channelling instabilities induced by iron diapirs, Earth Planet. Sci. Lett., 271, 24-33.

Golabek, G. J., T. V. Gerya, B. J. P. Kaus, R. Ziethe, and P. J. Tackley (2009), Rheological controls on the terrestrial core formation mechanism, G3, 10, $11,007-11,038$.

Hadamard, J. (1911), Mouvement permanent lent d'une sphère liquide et visqueuse dans un liquide visqueux, C. R. Acad. Sci., 152, 1735-1738.

Honda, R., H. Mizutani, and T. Yamamoto (1993), Numerical simulation of Earth's core formation., J. Geophys. Res., 98, 2075-2090.

Hood, L. L., N. C. Richmond, E. Pierazzo, and P. Rochette (2003), Distribution of crustal magnetic fields on Mars: Shock effects of basin-forming impacts, Geophys. Res. Lett., 30(6), 1-4.

Jacobsen, S. B. (2005), The Hf-W Isotopic System and the Origin of the Earth and Moon, Ann. Rev. of Earth and Planet. Sci., 33, 531-570.

Johnson, C. L., and R. J. Phillips (2005), Evolution of the Tharsis region of Mars: insights from magnetic field observations, Earth and Planet. Sci. Lett., 230, 241-254

Kaula, W. M. (1979), Thermal evolution of earth and moon growing by planetesimal impacts, J. Geophys. Res., 84, 999-1008.

Ke, Y., and V. S. Solomatov (2009), Coupled core-mantle thermal evolution of early Mars, J. Geophys. Res. (Planets), 114(13), 1-12.

Kleine, T., C. Münker, K. Mezger, and H. Palme (2002), Rapid accretion and early core formation on asteroids and the terrestrial planets from HfW chronometry, Nature, 418, 952-955. 
681 Kokubo, E., and S. Ida (1996), On runaway growth of planetesimals, Icarus,

682 123, 180-191.

Kuang, W., and J. Bloxham (1997), An Earth-like numerical dynamo model, Nature, 389, 371-374.

Labrosse, S., J. Poirier, and J. Le Mouel (1997), On cooling of the Earth's core, Phys. of the Earth and Planet. Int., 99, 1-17.

Laney, C. B. (1998), Computational gasdynamics, Cambridge University Press, Cambridge.

Lawrence, K., C. Johnson, L. Tauxe, and J. Gee (2008), Lunar paleointensity measurements: Implications for lunar magnetic evolution, Phys. of the Earth and Planet. Int., 168, 71-87.

Lillis, R. J., H. V. Frey, and M. Manga (2008), Rapid decrease in Martian crustal magnetization in the Noachian era: Implications for the dynamo and climate of early Mars, Geoph. Res. Lett., 35, 14,203-14,209.

Monteux, J., N. Coltice, F. Dubuffet, and Y. Ricard (2007), Thermo-mechanical adjustment after impacts during planetary growth, Geophys. Res. Lett., 34, 24 201-24 205.

Monteux, J., Y. Ricard, N. Coltice, F. Dubuffet, and M. Ulvrova (2009), A model of metal-silicate separation on growing planets, Earth and Planet. Sci. Lett., 287, 353-362.

Nimmo, F. (2009), Energetics of asteroid dynamos and the role of compositional convection, Geoph. Res. Lett., 36, 10,201-10,207.

Nimmo, F., and D. J. Stevenson (2000), Influence of early plate tectonics on the thermal evolution and magnetic field of Mars, J. Geophys. Res., 105, $11,969-11,980$. 
O'Keefe, J. D., and T. J. Ahrens (1977), Impact-induced energy partitioning, melting, and vaporization on terrestrial planets, in Lun. Planet. Sci. Conf., vol. 8, edited by R. B. Merril, pp. 3357-3374.

Peaceman, D. W., and H. H. Rachford (1955), The numerical solution of parabolic and elliptic differential equations, J. Soc. Ind. Appl. Math., 3, 2841.

Pierazzo, E., A. M. Vickery, and H. J. Melosh (1997), A Reevaluation of Impact Melt Production, Icarus, 127, 408-423.

Ratcliff, J. T., P. J. Tackley, G. Schubert, and A. Zebib (1997), Transitions in thermal convection with strongly variable viscosity, Phys. Earth Planet. Int., 102, 201-212.

Reese, C. C., and V. S. Solomatov (2006), Fluid dynamics of local martian magma oceans, Icarus, 184, 102-120.

Reese, C. C., and V. S. Solomatov (2010), Early martian dynamo generation due to giant impacts, Icarus, 20\%, 82-97.

Ricard, Y., O. Srámek, and F. Dubuffet (2009), A multi-phase model of runaway core-mantle segregation in planetary embryos, Earth and Planet. Sci. Lett., 284, 144-150.

Roberts, J. H., R. J. Lillis, and M. Manga (2009), Giant impacts on early Mars and the cessation of the Martian dynamo, J. Geophys. Res. (Planets), $114(13)$, 4009-4019.

Roe, P. L. (1986), Characteristic-based schemes for the Euler equations, Annual Review of Fluid Mechanics, 18, 337-365. 
Rubie, D. C., H. J. Melosh, J. E. Reid, C. Liebske, and K. Righter (2003), Mechanisms of metal-silicate equilibration in the terrestrial magma ocean, Earth and Planet. Sci. Lett., 205, 239-255.

Rybczynski, W. (1911), über die fortschreitende bewegung einer flüssigen kugel in einen medium, Bull. Acad. Sci. Cracovie, 1, 40-46.

Safronov, V. S. (1978), The heating of the earth during its formation, Icarus, 33, 3-12, doi:10.1016/0019-1035(78)90019-2.

Samuel, H., and P. J. Tackley (2008), Dynamics of core formation and equilibration by negative diapirism, Geochem. Geophys. Geosyst., 9, 6011-6026.

Samuel, H., P. J. Tackley, and M. Evonuk (2010), Heat partitioning in terrestrial planets during core formation by negative diapirism, Earth and Planet. Sci. Lett., 290, 13-19.

Schubert, G., C. T. Russell, and W. B. Moore (2000), Geophysics: Timing of the Martian dynamo, Nature, 408, 666-667.

Schubert, G., D. L. Turcotte, and P. Olson (2001), Mantle convection in the Earth and planets, Cambridge University Press.

Senshu, H., K. Kuramoto, and T. Matsui (2002), Thermal evolution of a growing Mars, J. Geophys. Res., 10\%, 1-13.

Shannon, M. C., and C. B. Agee (1996), High pressure constraints on percolative core formation, Geophys. Res. Lett., 23, 2717-2720.

Solomatov, V. S. (2000), Fluid Dynamics of a Terrestrial Magma Ocean, pp. 323-338, Origin of the earth and moon, edited by R.M. Canup and K. Righter and 69 collaborating authors. Tucson: University of Arizona Press., p.323-338. 
Srámek, O., Y. Ricard, and F. Dubuffet (2010), A multiphase model of core formation, G. J. Int., 181, 198-220.

Stegman, D. R., A. M. Jellinek, S. A. Zatman, J. R. Baumgardner, and M. A. Richards (2003), An early lunar core dynamo driven by thermochemical mantle convection, Nature, 421, 143-146.

Stevenson, D. J. (1990), Fluid dynamics of core formation, 231-249 pp., Origin of the Earth edited by H. E. Newsom and J. H. Jones, eds., Oxford Univ., New York.

Stevenson, D. J. (2003a), Planetary magnetic fields, Earth and Planet. Sci. Lett., 208, 1-11.

Stevenson, D. J. (2003b), Planetary science: Mission to Earth's core - a modest proposal, Nature, 423, 239-240.

Stevenson, D. J., T. Spohn, and G. Schubert (1983), Magnetism and thermal evolution of the terrestrial planets, Icarus, 54, 466-489.

Tonks, W. B., and H. J. Melosh (1992), Core formation by giant impacts, Icarus, 100, 326-346.

Touboul, M., T. Kleine, B. Bourdon, H. Palme, and R. Wieler (2007), Late formation and prolonged differentiation of the Moon inferred from $\mathrm{W}$ isotopes in lunar metals, Nature, 450, 1206-1209.

Turner, J. S. (1973), Buoyancy Effects in Fluids, Cambridge: Cambridge University Press.

Weiss, B. P., J. S. Berdahl, L. Elkins-Tanton, S. Stanley, E. A. Lima, and L. Carporzen (2008), Magnetism on the Angrite Parent Body and the Early Differentiation of Planetesimals, Science, 322, 713-716. 
776 Williams, J., and F. Nimmo (2004), Thermal evolution of the Martian core:

777 Implications for an early dynamo, Geology, 32.

778 Wood, B. J., M. J. Walter, and J. Wade (2006), Accretion of the Earth and 779 segregation of its core, Nature, 441, 825-833.

780 Yin, Q., S. B. Jacobsen, K. Yamashita, J. Blichert-Toft, P. Télouk, and F. Al781 barède (2002), A short timescale for terrestrial planet formation from Hf-W 782 chronometry of meteorites, Nature, 418, 949-952.

${ }_{783}$ Yoshino, T., M. J. Walter, and T. Katsura (2003), Core formation in planetesi784 mals triggered by permeable flow, Nature, 422, 154-157. Zerr, A., and R. Boehler (1994), Constraints on the melting temperature of the lower mantle from high-pressure experiments on $\mathrm{MgO}$ and magnesioüstite, ${ }_{787} \quad$ Nature, 371, 506-508, doi:10.1038/371506a0.

Ziethe, R., and T. Spohn (2007), Two-dimensional stokes flow around a heated cylinder: A possible application for diapirs in the mantle, J. Geophys. Res., 112, 1-13. 
Figure 1: Schematic view of the chemical equilibration following a large impact on an undifferentiated protoplanet. Within the isobaric region that results from the dissipation of the shock wave (time step $t_{1}$ ), the temperature increase (orange circle, $t_{2}$ ) melts the metal (red) that segregates rapidly from silicates (green) $\left(t_{3}\right)$. The initial position of the inertial centre of the iron diapir is indicated with a black dot (i.c.). Then the metallic phase sinks by a diapiric instability $\left(\mathrm{t}_{4}\right)$. Subsequent convection within the protocore can initiate an early magnetic field $\left(t_{5}\right)$. The top left panel shows where viscous heating $\left(H_{v}\right)$ occurs for a spherical density anomaly (i.e. a metallic protocore) outlined by the white dashed line within a RybczynskiHadamard analytical velocity field (see text for details). $H_{v} \sim \underline{\varepsilon}^{2}$ where $\underline{\varepsilon}$ is the dimensionless strain rate tensor. The viscous heating is shown for 2 viscosity ratios, $\lambda$, between the hot protocore and the cold undifferentiated material. For $\lambda=1$ (uniform viscosity), viscous heating can occur within the sphere, while for $\lambda<<1$, viscous heating only occurs outside the sphere. Here, $H_{v}$ is normalized by the maximum value of $H_{v}$ when $\lambda=1$.

Figure 2: Initial temperature of the protocore once at the centre of the planet as a function of the planetary radius. Each symbol represents a numerical result for a given $R_{F e}\left(R_{F e}\right.$ in the range $150-500 \mathrm{~km}$ ) for a uniform viscosity. Theoretical predictions for $T_{\text {core }}$ from Eq.14 are given for uniform viscosity (black solid line, $a(\lambda)=15 \%$ ) and temperature dependent viscosity (black dashed line, $a(\lambda)=0 \%$ ). The black solid line is without melting and the grey one is with melting. The region in between defines the range of initial protocore temperature (see Eq.5). The dotted line shows $T=T_{0}$. The chondritic solidus (Agee, 1997) and the dry peridotite solidus (Zerr and Boehler, 1994) at $P=P_{C M B}$ are represented with dashed-double-dotted lines (see section 5.2 for calculation details).

Figure 3: Temporal evolution (symbols from black to white) of the ratio $Q / \Phi$ as a function of the planetary radius. For each planetary radius, we investigated several diapir sizes at times $t=1$ and $50 \mathrm{Myr}$ for a uniform viscosity. The length of the rectangles represents the variation of $Q / \Phi$ for the range of diapir sizes. The width of each rectangle is arbitrary. Theoretical predictions for $\left(Q_{\text {cond }} / \Phi\right)$ at $t=0$ Myr from Eq.24 for uniform viscosity (black dashed line, $a(\lambda)=15 \%$ ) and temperature dependent viscosity (black dotted line, $a(\lambda)=0 \%$ ) are shown. Planetary and moon radii of small solar system objects are indicated on the top x-axis. 
Table 1: Typical parameter values for numerical models

\begin{tabular}{|c|c|c|}
\hline Planet radius & $R$ & $2000-4000 \mathrm{~km}$ \\
\hline Diapir (protocore) radius & $R_{F e}$ & $150-500 \mathrm{~km}$ \\
\hline Density difference & $\Delta \rho_{0}$ & $4500 \mathrm{~kg} \mathrm{~m}^{-3}$ \\
\hline Average density & $\rho_{0}$ & $4270 \mathrm{~kg} \mathrm{~m}^{-3}$ \\
\hline Iron density & $\rho_{F e}$ & $8000 \mathrm{~kg} \mathrm{~m}^{-3}$ \\
\hline Silicate density & $\rho_{S i}$ & $3500 \mathrm{~kg} \mathrm{~m}^{-3}$ \\
\hline Mean thermal expansion & $\alpha$ & $4.4 \times 10^{-5} \mathrm{~K}^{-1}$ \\
\hline Iron thermal expansion & $\alpha_{F e}$ & $1.5 \times 10^{-5} \mathrm{~K}^{-1}$ \\
\hline Silicate thermal expansion & $\alpha_{S i}$ & $5 \times 10^{-5} \mathrm{~K}^{-1}$ \\
\hline Iron heat capacity & $C_{p, F e}$ & $800 \mathrm{~J} \mathrm{~K}^{-1} \mathrm{~kg}^{-1}$ \\
\hline Silicate heat capacity & $C_{p, S i}$ & $1000 \mathrm{~J} \mathrm{~K}^{-1} \mathrm{~kg}^{-1}$ \\
\hline Average specific heat & & \\
\hline of the impacted body & $\overline{\rho_{0} C_{p}}$ & $4 \times 10^{6} \mathrm{~J} \mathrm{~K}^{-1} \mathrm{~m}^{-} 3$ \\
\hline Initial temperature & $T_{0}$ & $1250 \mathrm{~K}$ \\
\hline Iron melting temperature & $T_{m e l t, F e}$ & $1250 \mathrm{~K}$ \\
\hline Silicate melting temperature & $T_{m e l t, S i}$ & $1650 \mathrm{~K}$ \\
\hline Iron latent heat & $L_{F e}$ & $4 \times 10^{5} \mathrm{~J} \mathrm{~kg}^{-1}$ \\
\hline Silicate latent heat & $L_{S i}$ & $2.7 \times 10^{5} \mathrm{~J} \mathrm{~kg}^{-1}$ \\
\hline Thermal diffusivity & $\kappa$ & $10^{-6} \mathrm{~m}^{2} \mathrm{~s}^{-1}$ \\
\hline Mean thermal conductivity & $k=k_{S i}$ & $4 \mathrm{~W} \mathrm{~m}^{-1} \mathrm{~K}^{-1}$ \\
\hline Iron thermal conductivity & $k_{F e}$ & $40 \mathrm{~W} \mathrm{~m}^{-1} \mathrm{~K}^{-1}$ \\
\hline Metal content & $f_{0}$ & 0.17 \\
\hline Reference viscosity & $\eta_{0}$ & $10^{22} \mathrm{~Pa} \mathrm{~s}$ \\
\hline Impact energy conversion & & \\
\hline coefficient & $\gamma$ & $0.3-0.5$ \\
\hline Volume effectively heated & & \\
\hline by impact & $h_{m}$ & 2.7 \\
\hline Gravitational constant & G & $6.67 \times 10^{-11} \mathrm{~m}^{3} \mathrm{~kg}^{-1} \mathrm{~s}^{-2}$ \\
\hline Average magnetic field strength & $\bar{B}$ & $2.5 \mathrm{mT}$ \\
\hline Magnetic diffusivity & $\nu$ & $2 \mathrm{~m}^{2} \mathrm{~s}^{-1}$ \\
\hline Magnetic permeability & $\mu$ & $4 \pi \times 10^{-7} \mathrm{H} \mathrm{m}^{-1}$ \\
\hline
\end{tabular}


Figure 4: $Q / \Phi 1 \mathrm{Myr}$ after the diapir reaches the center of the impacted planet as a function of the metallic diapir radius (black symbols). The protocore radius differentiated after a single impact corresponds to an impactor radius indicated on the top $\mathrm{x}$-axis. The symbol shape is related to the radius of the impacted planet. The black dashed line represents the threshold expression from Eq.25 in the isoviscous case $(a(\lambda)=15 \%)$. Above this dashed line (white domain) a dynamo is plausible, while below it no dynamo is generated. The dotted line corresponds to the same threshold but assuming a protocore less viscous than the surrounding mantle $(a(\lambda)=0 \%)$. If $Q / \Phi$ after $50 \mathrm{Myr}$ is within the dynamo domain, a second white filled symbol represents $Q / \Phi$ at this time.

Figure 5: Magnetic Reynolds number of the protocore as a function of its size from our numerical models. The protocore radius differentiated after a single impact corresponds to an impactor radius indicated on the top x-axis. The symbol shape is related to the radius of the impacted planet and its color (black or white) to the time after the diapir reaches the center of the impacted planet. The dotted line represents the numerical results expected from a temperature-dependent viscosity model (i.e., $R e_{m}$ from isoviscous models divided by a factor 1.4). The theoretical Reynolds number values obtained from Eq.21 are represented with a solid line (with $\Delta T_{0}$ corresponding to $\mathrm{R}=4000 \mathrm{~km}$ ) and a dashed line (with $\Delta T_{0}$ corresponding to $\mathrm{R}=2000 \mathrm{~km}$ ). The grey domain illustrates $R e_{m}^{\text {crit }}$ above which a dynamo is plausible.

Figure 6: Time to remove the excess heat from the protocore for 3 different protocore-mantle boundary heat transfers as a function of the planetary radius. The dotted line represents the case of a conductive boundary layer (Eq.22, $\delta=R_{F e}$ and Eq.27) and the dashed line represents the case of a time dependent conductive boundary layer (Eq.22, $\delta=2 \sqrt{\kappa t}$ and Eq.27). The black line shows the case where a molten layer surrounds the protocore (Eq.23, $\eta_{m e l t}=100$ Pa.s and Eq.27). This time also depends on the size of the protocore. For each case, 2 lines delimit a domain from $R_{F e}=150 \mathrm{~km}$ (lower line) to $500 \mathrm{~km}$ (upper line). 